\title{
Estimation of mating system parameters in an evolving gynodioecous population of cultivated sunflower (Helianthus annuus L.)
}

\author{
M Roumet, M-F Ostrowski, J David, C Tollon and M-H Muller
}

Cultivated plants have been molded by human-induced selection, including manipulations of the mating system in the twentieth century. How these manipulations have affected realized parameters of the mating system in freely evolving cultivated populations is of interest for optimizing the management of breeding populations, predicting the fate of escaped populations and providing material for experimental evolution studies. To produce modern varieties of sunflower (Helianthus annuus L.), self-incompatibility has been broken, recurrent generations of selfing have been performed and male sterility has been introduced. Populations deriving from hybrid-F1 varieties are gynodioecious because of the segregation of a nuclear restorer of male fertility. Using both phenotypic and genotypic data at 11 microsatellite loci, we analyzed the consanguinity status of plants of the first three generations of such a population and estimated parameters related to the mating system. We showed that the resource reallocation to seed in male-sterile individuals was not significant, that inbreeding depression on seed production averaged $15-20 \%$ and that cultivated sunflower had acquired a mixed-mating system, with $\sim 50 \%$ of selfing among the hermaphrodites. According to theoretical models, the female advantage and the inbreeding depression at the seed production stage were too low to allow the persistence of male sterility. We discuss our methods of parameter estimation and the potential of such study system in evolutionary biology.

Heredity (2012) 108, 366-374; doi:10.1038/hdy.2011.79; published online 14 September 2011

Keywords: sunflower; mating system; gynodioecy; population genetics; crop evolution

\section{INTRODUCTION}

Cultivated plants are the product of the adaptation of wild species to cultivation and use by humans. Domestication resulted in dramatic changes in morphological (for example, plant architecture), biochemical and life-history traits (seed dormancy, phenology, mating systems; Gross and Olsen, 2010) and made crops outstanding study systems for investigating the genetic bases of adaptation (Ross-Ibarra et al., 2007). Moreover, a great deal of genetic mechanisms involved for instance in the resistance to pathogens or in different aspects of plant reproduction (self-incompatibility, restoration of male fertility) have been first unraveled in crop plants (Hancock, 2005). Reciprocally, when human control is at least partially relaxed, populations of cultivated plants are confronted with a new set of evolutionary forces and can adapt quite quickly to new environmental conditions. This is exemplified by the recurrent evolution of weeds from escaped cultivated plants (Ellstrand et al., 2010), or by dynamic management experiments where artificial populations of crops have been placed in contrasted environments (for example, differentiation of heading time after 10 generations among populations of dynamic management of wheat; Goldringer et al., 2006).

Mating system has been affected by domestication and the subsequent improvement of cultivated plants (Glémin and Bataillon, 2009). Recently, it has been the target of intentional manipulations by plant breeders, especially when shifting from heterogeneous landraces and mass selection to very specific selection schemes such as production of inbred lines in outcrossing species or hybrid-F1. Questions arise as to how these manipulations affected the realized mating system and traits related to it in cultivated plants. Addressing such issues is of interest to crop science. It may help optimizing the management of breeding populations by improving the conservation of genetic diversity and evolutionary potential. It is also a way to investigate the evolutionary consequences of crop escape into the wild and to predict the impact of the decrease of pollinator abundance on seed production (Eckert et al., 2010). Moreover, from an evolutionary perspective, cultivated populations could provide material for experimental evolution designs to investigate the correlated responses triggered out by transitions in mating system (for example, increase of pollen production in outcrossed population of wheat; David and Pham, 1993).

Sunflower (Helianthus annuus L.) is an annual, self-incompatible, bee-pollinated plant, domesticated around 4000 BP in North America (Putt, 1997). Until the second part of the twentieth century, sunflower was cultivated as free-pollinated landraces and remained strictly selfincompatible. In order to produce inbred lines and to reduce the dependence on pollinators for seed production under field conditions, breeders have recently broken the self-incompatibility system and selected self-fertility in cultivated varieties (Gandhi et al., 2005). Moreover, to facilitate the production of hybrid-F1 varieties, breeders also introduced a male-sterile cytoplasmic sterility system from the related species Helianthus petiolaris (Rieseberg et al., 1994): F1-hybrids 
are obtained through the cross of maternal male-sterile lines (carrying male sterility cytotype (CMS) and nuclear recessive non-restorer alleles $r f / r f$ ) with paternal restorer lines (carrying a fertile cytoplasm and dominant restorer alleles: $R F / R F$ ). The breeding system of this recently self-compatible species in natural conditions is unknown: there are currently no data on the realized outcrossing rates or on the actual inbreeding depression. Similarly, whether the introduction of genetic male sterility was associated with the reallocation of resources no longer used for pollen production to other fitness components in male-sterile individuals (that is, female compensation or female advantage; Shykoff et al., 2003) remains an important and open question.

In this study, we used a population derived from a F1-hybrid variety to estimate parameters of the mating system in cultivated sunflower. Interestingly, such population can be analyzed in the frame of models of gynodioecy evolution. F1-hybrids are hermaphrodite: they carry the CMS cytotype and are heterozygous at the nuclear restorer locus. When they reproduce, the segregation at the restorer locus leads to the coexistence of male-sterile and hermaphrodite individuals and thus to a gynodioecious population. Gynodioecy has long been of considerable interest to evolutionary biologists (for a review, see Shykoff et al., 2003). Wealth of models have been designed to explain the persistence of females, who cannot gain any fitness through pollen, contrary to the hermaphrodites. When sex determination is nuclear (as in the present population), models predict that females can spread in populations only if they are more than twice as fecund as hermaphrodites. This higher female fitness can result from the reallocation of resources through female compensation and/or from the avoidance of the inbreeding depression that can affect the selfed offspring of hermaphrodites (Charlesworth and Charlesworth, 1978).

Following these models and using phenotypic and microsatellite data gathered over three generations in our hybrid-F1 population, we estimated the mating system parameters that are expected to determine the fate of male sterility in the population, that is, the outcrossing rate, the inbreeding depression on seed production and the female compensation. We incorporated our estimates into the model of gynodioecy evolution developed by Charlesworth and Charlesworth (1978) and predicted the fate of male-sterile individuals in this population. Finally, we discuss how our estimation procedures and plant material might be useful for further studies of mating system evolution.

\section{MATERIALS AND METHODS}

\section{Experimental population}

In 2001, the F1-hybrid variety Prodisol (DEKALB) was cultivated in a 0.6 ha field (INRA Experimental Station, Epoisses, France). The descent of this variety was harvested and called generation g2. In 2002, soybean was sown on this field and volunteer sunflowers appeared spontaneously at a density ranging from 0.5 to 4 plants per $\mathrm{m}^{2}$. These volunteers originated from seeds lost at harvest and were representative of generation g2. The descent of 335 of these plants was collected in bulk and made up generation g3. During these 2 years, there was no other sunflower field at a distance of $<400 \mathrm{~m}$ from our field. In 2004, 350 seeds of generation g3 were sown under a pollen-proof tunnel (INRA, Mauguio, France). Honeybees were introduced during the flowering period to ensure free intercrossing of the plants. The descent of these plants was bulked and constituted generation $\mathrm{g} 4$.

This situation-isolated population, non-overlapping generations, nuclear sex determinism and partially self-fertilizing hermaphrodites-matches the model described in Charlesworth and Charlesworth (1978). According to this model, sex ratio evolution depends on three parameters: $k$, the female advantage; $\delta$ the inbreeding depression; and $t_{\mathrm{h}}$ the hermaphrodite outcrossing rate (Table 1). Figure 1 illustrates how these parameters are involved in the evolution of adult sex ratio.

\section{Table 1 Definition of the parameters}

\begin{tabular}{ll} 
Symbol & Definition \\
\hline$k$ & $\begin{array}{l}\text { Female advantage. }(1+k) \text { is the amount of seed produced by females } \\
\text { relative to that of hermaphrodites } \\
\text { Inbreeding depression. }(1-\delta) \text { corresponds to the number of viable off- } \\
\text { springs produced by individuals originating from selfing relative to that } \\
\text { of individuals originating from outcrossing. }\end{array}$ \\
& $\begin{array}{l}\text { Population-level outcrossing rate; the proportion of outcrossed seeds in } \\
\text { the population } \\
t_{g}\end{array} \quad \begin{array}{l}\text { Population-level outcrossing rate between generation } g \text { and } g+1 \\
t_{h}\end{array} \quad \begin{array}{l}\text { Outcrossing rate of the hermaphrodites } \\
t_{h g}\end{array}$ \\
& $\begin{array}{l}\text { Outcrossing rate of the hermaphrodites between generation } g \text { and } g+1: \\
\text { the proportion of outcrossed seeds in the progeny of a hermaphrodite. }\end{array}$ \\
\end{tabular}

\section{Experimental design and data production}

In April 2006, seeds (that is, achenes) from each generation (g2, g3 and g4) were sown in an experimental field (Mauguio, France). The field was divided into three blocks of 3.25 by $8.40 \mathrm{~m}$, each devoted to a single generation. Blocks were made of five plots separated from one another by $1.20 \mathrm{~m}$. Each plot contained two rows where one to three seeds were sown every $25 \mathrm{~cm}$. A total of 217, 189 and 247 individuals, respectively, emerged in the g2, g3 and g4 blocks. Block, plot, row and position on the row were recorded for each individual. Portions of leaves were sampled from every g3 and g4 plant.

During summer 2006, the sexual morph and three measures of seed production (total seed weight, total seed number and seed size) were recorded for each plant. Seed size was calculated as the total seed weight divided by the total seed number. Before the analysis, total seed weight and seed size were square-root transformed and total seed number was log transformed to satisfy the assumptions of the analysis of variance.

DNA was isolated from $\sim 100 \mathrm{mg}$ of plant leaves according to the Dneasy Plant Mini kit protocol (Qiagen, GmbH, Hilden, Germany) with the following modification: $1 \%$ of Polyvinylpyrrolidone $(40000)$ was added to buffer AP1. Because of DNA quality problems, only 126 and 216 individuals from generation g3 and g4, respectively, were genotyped at 11 microsatellite loci separated by at least $36 \mathrm{cM}$ : ORS297, ORS303, ORS309, ORS371, ORS380, ORS432, ORS610, ORS788, ORS887, ORS925 and ORS1085 (Tang et al., 2002). The amplification reaction consisted of $50 \mathrm{ng}$ DNA, 4 pmol of unlabeled reverse primer and 2 pmol of forward primer, fluorescently labeled with NED, HEX or FAM, $1 \times$ reaction buffer, $2 \mathrm{~mm} \mathrm{MgCl}_{2}, 200 \mu \mathrm{M}$ dNTP and $0.25 \mathrm{U}$ Taq DNA polymerase, in a total volume of $25 \mu$ l. The amplification method was as follows: $95^{\circ} \mathrm{C}$ for $2 \mathrm{~min}, 36$ cycles of $94^{\circ} \mathrm{C}$ for $30 \mathrm{~s}, T_{x}$ for $30 \mathrm{~s}$ ( $T_{x}$ was initially $63{ }^{\circ} \mathrm{C}$ and decreased of $1{ }^{\circ} \mathrm{C}$ per cycle for the 6 first cycles, until it reached $57^{\circ} \mathrm{C}$ ), and $72{ }^{\circ} \mathrm{C}$ for $45 \mathrm{~s}$; followed by a final extension for $20 \mathrm{~min}$ at $72^{\circ} \mathrm{C}$. Electrophoresis was performed on an ABI 3130xl Genetic Analyser (Applied Biosystems, Foster City, CA, USA). Samples were prepared by adding $3 \mu \mathrm{l}$ of diluted PCR products to $6.875 \mu \mathrm{l}$ formamide and $0.125 \mu \mathrm{l}$ of GenScan 400HD Rox size standard (Applied Biosystems). The GENEMAPPER software (Applied Biosystems) was used to analyze the DNA fragments and to score the genotypes.

\section{Impact of competition on seed production}

The rows contained a variable number of plants, possibly creating local variation in competition intensity. To account for this, we built a variable (C) describing the competition experienced by each plant, assuming that: (1) the competition between two individuals $i$ and $j$ is inversely proportional to the distance $d_{i j}$ between them; (2) the competition experienced by an individual $i$ is the sum of the competition with all the individuals surrounding $i$; and (3) if the distance between two individuals $i$ and $j$ exceeds $d_{i j \max }$, the competition between these individuals is null. Accordingly, $C$ was defined as:

$$
C_{i}=\sum_{j=1}^{J} \frac{1}{d_{i j}}
$$




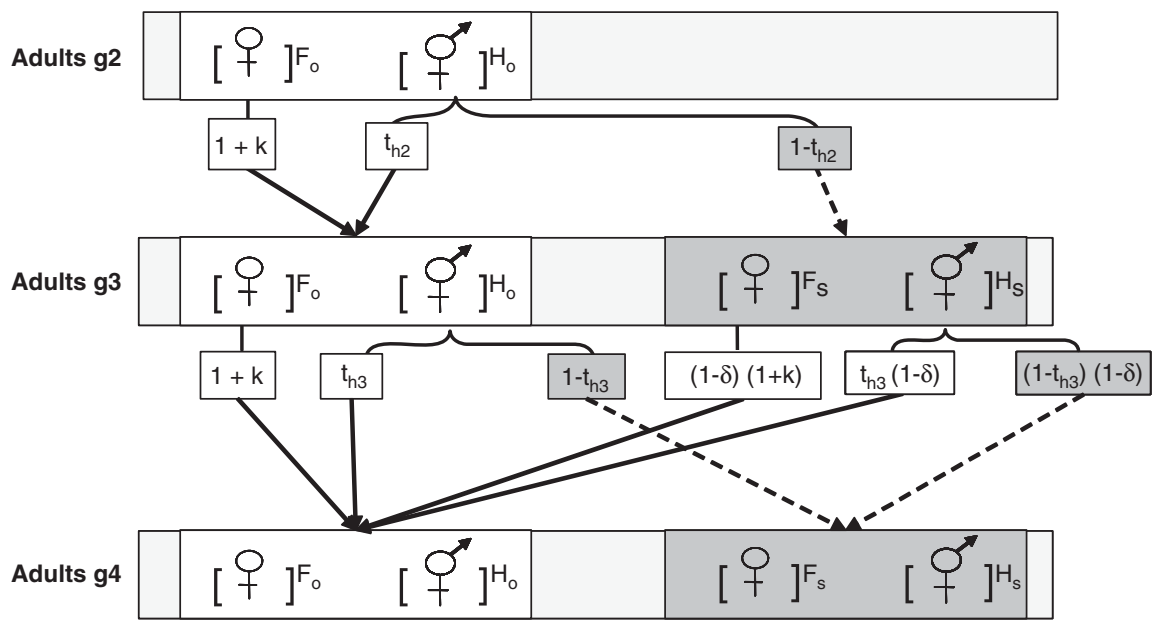

Figure 1 Schematic representation of the reproduction process in the first generations of the experimental population. The subscripts $F_{O}$ and $F_{S}$ denote the outcrossed vs selfed origin of females; $H_{O}$ and $H_{S}$ the outcrossed vs selfed origin of hermaphrodites. Dotted arrows represent reproduction by selfing; solid arrows represent reproduction by outcrossing. See Table 1 for the definitions of parameters.

Table 2 ANCOVA testing the effects of competition and generation on total seed weight

\begin{tabular}{lrrrr}
\hline & d.f. & \multicolumn{1}{c}{ SS } & \multicolumn{1}{c}{$F$} & P-value \\
\hline Competition & 1 & 366.58 & 86.4518 & $<0.001$ \\
Generation & 2 & 21.54 & 2.5404 & 0.09 \\
Competition $\times$ generation & 2 & 3.98 & 0.4688 & 0.63 \\
Residuals & 511 & 2166.79 & & \\
\hline
\end{tabular}

Abbreviations: ANCOVA, analysis of covariance; d.f., degrees of freedom; F, F-test statistic; SS, sum of squares.

where $d_{i j} \in\left[0 ; d_{i j \max }\right] ; d_{i j \max }$ was chosen to maximize the coefficient of determination $\left(R^{2}\right)$ of the regression of total seed weight (which represents the total investment in reproduction) on $C . R^{2}$ was maximum (19\%) for $d_{i j \max }=95 \mathrm{~cm}$.

Before the analysis, $C$ was $\log$ transformed. We performed an analysis of covariance (performed in R-2.5.1 (R Development Core Team, 2008) package lmtest) with generation as a fixed factor and $C$ as a covariate to check that the effect of competition on total seed weight was significant and similar for each generation (Table 2).

\section{Female advantage}

The effect of sexual morph on seed production was tested in a mixed model using the backward suppression of nonsignificant effects (R-2.5.1 package nlme). Factors included in the model were sexual morph, generation, plot, location on a row (border or central position) and competition. Sex, generation and location on a row were declared as fixed effects; plot was nested within generation and declared as a random effect. Competition was taken into account by adding the covariable $C$ to the model.

Based on model predictions we estimated the mean seed production of females and hermaphrodites and calculated the female advantage $(k)$ as the relative excess of female seed production compared with hermaphrodites.

\section{Genetic diversity and effective population size}

The number of alleles and Nei's expected heterozygosity $\left(H_{\mathrm{e}}, \mathrm{Nei}, 1987\right)$ were computed for each generation using GENETIX (Belkhir et al., 2001).

The effective population size $N_{\mathrm{e}}$ was estimated using the temporal variation of allelic frequencies at the microsatellite loci between g3 and g4. We used Waples' estimator (1989) based on $F_{\mathcal{C}}$, the standardized variance in allelic frequencies between sampled generations. The $95 \%$ confidence interval on $N_{\mathrm{e}}$ was obtained using simulations of the actual distribution of $F_{\mathrm{c}}$ based on the estimated $N_{\mathrm{e}}$, as suggested by Goldringer and Bataillon (2004). This method was implemented in a program kindly provided by M Siol and described in Siol et al. (2007). Rare alleles present in only one or two individuals over the whole data set were pooled.

\section{Inference on individual consanguinity status based on multilocus} genotypes

Each individual could be assigned to a class of inbreeding $S_{n}$ with $n$ the number of selfing generations since the last outcrossing event in its genealogy $(0 \leqslant n \leqslant \infty)$. As our initial population is a F1-hybrid variety made up of a unique genotype, $\mathrm{g} 2$ individuals resulting from outcrossing and selfing were genetically indistinguishable. We therefore considered that generation g2 included a single class $S_{0}$, g3 two classes, $S_{0}$ and $S_{1}$, and g4 three classes, $S_{0}, S_{1}$ and $S_{2}$.

At each generation of selfing, the heterozygosity resulting from the initial outcrossing event is halved. Therefore, we used the individual multilocus heterozygosity pattern $G_{x}$ to compute $P\left(S_{n} \mid G_{x}\right)$, the probability that an individual with genotype $G_{x}$ belongs to the class $S_{n}$ (see Appendix for details). The g3 genotyped individuals were thus characterized by the probability distribution $\left(P\left(S_{0} \mid G_{x}\right), P\left(S_{1} \mid G_{x}\right)\right)$ and $g 4$ individuals by $\left(P\left(S_{0} \mid G_{x}\right), P\left(S_{1} \mid G_{x}\right)\right.$, $\left.P\left(S_{2} \mid G_{x}\right)\right)$.

The coefficient of inbreeding $\left(f_{x}\right)$ is the probability that two alleles in an individual $x$ are identical by descent. This coefficient is $0,0.5$ and 0.75 for individuals originating from outcrossing $\left(S_{0}\right)$, one generation of selfing $\left(S_{1}\right)$ and two generations of selfing $\left(S_{2}\right)$, respectively. The probabilities $P\left(S_{n} \mid G_{x}\right)$ were used to approximate the individual inbreeding coefficients using:

$$
f_{x}=P\left(S_{1} \mid G_{x}\right) \times 0.5+P\left(S_{2} \mid G_{x}\right) \times 0.75 \text {. }
$$

\section{Estimation of population outcrossing rates}

Multilocus individual heterozygosities were also used to estimate population outcrossing rates following the method of Enjalbert and David (2000). The likelihood of a sample of $Y$ individuals of genotypes $\left(G_{1}, \ldots, G_{Y}\right)$ is the product of individual genotype probabilities that are functions of the selfing rates in the previous generations (see Appendix, equation (A3)) (Enjalbert and David, 2000).

$$
L\left(G_{1}, G_{2} \ldots G_{Y}\right)=\prod_{x=1}^{Y} P\left(G_{x}\right) .
$$

Using the molecular data collected in generation g3, we estimated $t_{2}$ as the value that maximized the likelihood function. Similarly, using the $\mathrm{g} 4$ molecular data, we estimated $t_{2}$ and $t_{3}$ as the values that jointly maximized the likelihood function. Confidence intervals were computed as described in Enjalbert and David (2000). 


\section{Estimation of hermaphrodite outcrossing rates}

Hermaphrodite outcrossing rates $\left(t_{h}\right)$ are not directly assessable from molecular data. However, at a given generation, they are related to the population's outcrossing rate $t$, to $H_{O}$ and $H_{S}$-the relative frequencies of adult hermaphrodites originating, respectively, from outcrossing and selfing-and to $F_{O}$ and $F_{S}$-the relative frequencies of adult females originating, respectively, from outcrossing and selfing (Figure 1); $t_{h}$ could then be deduced from equation (4). Because of the large uncertainties around population parameters, this computation was only meant as an approximation for descriptive purposes.

$$
t=\frac{t_{h}\left[H_{O}+(1-\delta) H_{S}\right]+(1+k)\left[F_{O}+(1-\delta) F_{S}\right]}{H_{O}+(1-\delta) H_{S}+(1+k)\left[F_{O}+(1-\delta) F_{S}\right]}
$$

The outcrossing rate $t_{h 2}$ was computed using the value of $t_{2}$ estimated from g3 molecular data, the value of $k$ estimated from $\mathrm{g} 2$ total seed weight data and the observed frequencies of females and hermaphrodites in g2 $\left(F_{O}\right.$ and $\left.H_{O}\right)$. The outcrossing rate $t_{h 3}$ was computed using the value of $t_{3}$ estimated from g4 molecular data, the values of $k$ and $\delta$ estimated from g 3 total seed weight data (see below for $\delta$ ) and the frequencies of females and hermaphrodites originating from outcrossing and selfing in $\mathrm{g} 3\left(F_{O}, H_{O}, F_{S}\right.$ and $\left.H_{S}\right)$. Because the inbreeding status of g3 individuals is only known as a probability $P\left(S_{0} \mid G_{x}\right)$, we computed the frequencies of outbred individuals as:

$$
F_{O}=F \times \sum_{f} P\left(S_{0} \mid G_{f}\right) \quad H_{O}=H \times \sum_{h} P\left(S_{0} \mid G_{h}\right)
$$

where $F$ and $H$ refer, respectively, to the observed frequencies of females and hermaphrodites in g3. $P\left(S_{0} \mid G_{f}\right)$ (respectively $P\left(S_{0} \mid G_{h}\right)$ ) is the probability that female $f$ (respectively hermaphrodite $h$ ) belongs to the inbreeding class $S_{0}$, given its genotype. The frequency of inbred females (respectively hermaphrodites) was then obtained as $F-F_{O}$ (respectively $H-H_{O}$ ).

\section{Impact of inbreeding on seed production}

Different methods were used to detect and measure the impact of inbreeding on seed production.

We first tested in each generation whether inbreeding and the inbreeding $\times$ competition interaction affect seed production. We used a multiple linear regression model in which seed production depended on competition $C$, inbreeding coefficient $f$ (equation (2)) and the inbreeding $\times$ competition interaction, as follows:

$$
Y_{i}=\mu+d . f_{i}+b . C_{i}+r . f_{i} \cdot C_{i}+\varepsilon_{i},
$$

where $Y_{i}$ is the seed production of individual $i ; \mu$ is the mean seed production without both competition or consanguinity; $b$ and $d$ are the global impacts of competition and inbreeding, respectively; $r$ corresponds to the inbreeding $\times$ competition interaction and $\varepsilon_{i}$ is the residual error term.

Second, we estimated inbreeding depression $(\delta)$ in $\mathrm{g} 3$ and $\mathrm{g} 4$ as the reduction in fitness of selfed compared with outcrossed individuals:

$$
\delta_{g}=1-\frac{W_{\text {self }(g)}}{W_{\text {out }(g)}}
$$

where $W_{\text {out }(g)}$ and $W_{\text {self(g) }}$ correspond to the mean fitness of outcrossed $\left(S_{0}\right.$ class) and selfed ( $S_{1}$ and $S_{2}$ class) individuals at generation $g$, respectively. Inbreeding depression was estimated here considering each seed production variable separately as a component of fitness.

Because the inbreeding status of individuals was only known as a set of probabilities, we resampled 3000 data sets for each generation to estimate $W_{\text {self }(g)}$ and $W_{\text {out }(g)}$. The resampling procedure associated each phenotypic value to an inbreeding class randomly chosen in the probability distribution characterizing the genotyped individual $\left(P\left(S_{0} \mid G_{x}\right), P\left(S_{1} \mid G_{x}\right), P\left(S_{2} \mid G_{x}\right)\right)$. The mean fitnesses $W_{\text {self }(g)}$ and $W_{\text {out }(g)}$ were estimated as the values predicted by equation (6) in the absence of competition $(\mathrm{Ci}=0)$ and $\delta_{g}$ was computed. The results over the resampled data sets were used to estimate the mean values of inbreeding depression in g3 and g4 and their 95\% confidence intervals (intervals containing 95\% of the values of $\delta_{g}$ ).

Finally, we compared the impact of one generation of selfing in g3 and g4 on the seed production. In each resampled data set generated above, we eliminated any g4 individual assigned to class $S_{2}$. We then used a mixed model to check that the effects of competition on seed production did not differ between generations or cross type (selfed or outcrossed). In this model, generation and cross type were declared as fixed effects and competition as a covariate. When neither the competition $\times$ generation interaction nor the competition $\times$ cross-type interaction were significant for any of the seed production variables (which was true in $90 \%$ of the data sets), we tested the cross-type $\times$ generation interaction. We report the proportion of data sets with a significant interaction effect.

\section{Prediction of the fate of male sterility in the population}

Theoretically, females should persist in our population if $1+k>2\left(1-\left(1-t_{h}\right) \delta\right)$ (Charlesworth and Charlesworth, 1978). Using mean estimated values of $t_{h}, k$ and $\delta$ in $\mathrm{g} 3$ and $\mathrm{g} 4$, we checked if this condition was fulfilled in our experimental population. We used the estimations of $\delta$ based on total seed weight (total investment in reproduction).

We then incorporated the estimated effective population size in simulations to take into account both selective and stochastic processes in the evolution of female frequencies over generations. We used an iterative procedure adapted from Ostrowski et al. (2010) to simulate the genotypic frequencies at the restorer locus ( $R F / R F, R F / r f, r f / r f)$ in the different classes of consanguinity $S_{0}, S_{1} \ldots S_{n}$ at each generation, following the scheme of Figure 1. The genotypic frequencies at generation $g+1$ were generated by (1) computing the frequencies at generation $g+1$ using the frequencies at generation $g$, and the values of $k, \delta$ and $t_{h g} ;(2)$ randomly sampling $N_{\mathrm{c}}$ genotypes in the multinomial frequency distribution obtained; and (3) dividing the resulting vector of genotype numbers by $N_{\mathrm{c}}$. The frequency of females at generation $g$ was computed in a sample of $E$ individual genotypes drawn from the simulated frequencies. $N_{\mathrm{c}}$ corresponds to the number of zygotes drawn to contribute to the next generation and is related to $N_{\mathrm{e}}$ through the following equation: $N_{\mathrm{c}}=N_{\mathrm{e}}+N_{\mathrm{e}}(1-t) /(2-(1-t))$

Simulations were performed for two purposes. We first aimed at comparing the observed female frequencies in g2, g3 and g4 to the expected values under three different evolutionary scenarios: (1) considering the estimated values of $k$, $\delta$ and $t_{h g}$; (2) setting $k$ and $\delta$ to zero; and (3) setting $k, \delta$ and the outcrossing rates to zero. For this purpose, we used the sample sizes corresponding to the actual data set. For each set of evolutionary parameters, 1000 simulations were performed using Mathematica (Wolfram, 1996). We thus obtained, for each scenario, the expected distribution of frequency of females at each generation (mean frequency and 95\% confidence interval).

Second, we wanted to predict the persistence of male sterility after 100 generations in our population (under scenario 1). To achieve this, we counted the proportion of simulations in which females disappeared after 100 generations of evolution (that is, where the frequency of genotype $\mathrm{rf} / \mathrm{rf}$ was 0 at generation 100)

\section{RESULTS}

\section{Female advantage}

Based on raw data, females and hermaphrodites presented a very similar seed production (Table 3 ).

Because none of the interactions between the factors were significant for any seed production variable, these interaction terms were excluded from the model. The final model indicated that the effect of the sexual morph on seed production was nonsignificant (Table 4). However, it predicted a slightly larger seed production for females. After summing over all generations, this female advantage was $\sim 5 \%$ for the three seed production variables.

\section{Molecular data}

As expected for a population derived from a F1-hybrid variety, the genetic diversity was low. Over the whole sample, we scored between two and four alleles per locus (2.5 on average), which denotes that the hybrid-F1 variety originally sown was not perfectly pure based on molecular markers, as observed previously (see Ostrowski et al., 2010). Nei's expected heterozygosity per locus $\left(H_{\mathrm{e}}\right)$ ranged from 0.20 to 0.67 
Table 3 Summary of the sample design and main characteristics of the three studied generations (g2, g3 and g4)

\begin{tabular}{|c|c|c|c|c|c|c|c|c|c|c|}
\hline & \multirow[t]{2}{*}{ Total number of plants $(\mathrm{N})$} & \multirow[t]{2}{*}{ Sample size for genetic analysis } & \multicolumn{2}{|c|}{ Frequency (\%) } & \multicolumn{2}{|c|}{ Total seed weight (g) } & \multicolumn{2}{|c|}{ Seed size (g) } & \multicolumn{2}{|c|}{ Total seed number } \\
\hline & & & $F$ & $H$ & $F$ & $H$ & $F$ & $H$ & $F$ & $H$ \\
\hline g3 & 189 & 126 & 26.9 & 73.1 & 48.1 & 51.9 & $5 \times 10^{-2}$ & $4.9 \times 10^{-2}$ & 989.3 & 1036.9 \\
\hline g4 & 247 & 216 & 18 & 82.0 & 43.1 & 38.9 & $4.8 \times 10^{-2}$ & $4.3 \times 10^{-2}$ & 919.2 & 850.4 \\
\hline Total & 653 & 342 & 21 & 79 & 47.7 & 46.1 & $5 \times 10^{-2}$ & $4.7 \times 10^{-2}$ & 964.6 & 956.1 \\
\hline
\end{tabular}

For each generation, we indicate the number of plants $(N)$, the number of genotyped plants, the frequency and the mean seed production of females $(F)$ and hermaphrodites $(H)$.

Table 4 ANOVA (analysis of variance) testing the effects of sexual morph, generation, competition, plot and location on seed production variables

\begin{tabular}{|c|c|c|c|c|c|c|c|c|}
\hline \multirow[t]{2}{*}{ Source } & \multirow[t]{2}{*}{ Type } & \multirow[t]{2}{*}{ d.f. } & \multicolumn{2}{|c|}{ Total seed weight } & \multicolumn{2}{|c|}{ Seed size } & \multicolumn{2}{|c|}{ Total seed numbe } \\
\hline & & & $F / L R$ & $\mathrm{P}$-value & $F / L R$ & $\mathrm{P}$-value & $F / L R$ & P-value \\
\hline Plot (generation) & Random & 12 & 3.94 & 0.05 & 6.08 & $<0.05$ & 1.32 & 0.24 \\
\hline Generation & Fixed & 2 & 4.18 & $<0.05$ & 0.74 & 0.51 & 1.55 & 0.64 \\
\hline Competition & Fixed & 1 & 86.42 & $<0.001$ & 59.8 & $<0.001$ & 39.95 & $<0.001$ \\
\hline Location & Fixed & 1 & 5.65 & $<0.05$ & 13.51 & $<0.001$ & 0.31 & 0.57 \\
\hline Sexual morph & Fixed & 1 & 0.76 & 0.38 & 3.98 & 0.05 & 0.21 & 0.21 \\
\hline
\end{tabular}

F-value and likelihood ratio statistic (LR) are given for fixed and random effects, respectively.

(0.46 on average). The effective population size between g3 and g4 was 37.2 (95\% confidence interval: 14-95) that corresponds to the usual $N_{\mathrm{e}} / N$ ratio of $10 \%$ (Frankham, 1995; Ostrowski et al., 2010).

Using individual heterozygosity patterns, we computed in each generation the probability that an individual with genotype $G_{x}$ belongs to the inbreeding class $S_{n}\left(P\left(S_{n} \mid G_{x}\right)\right)$. The distributions of individual probabilities $P\left(S_{n} \mid G_{x}\right)$ are given in Figure 2. In g3, 65\% of individuals had a probability above $80 \%$ of belonging to a given class. In contrast, only $7 \%$ of $\mathrm{g} 4$ individuals had a probability $P\left(S_{n} \mid G_{x}\right)$ above $80 \%$, which means that the inbreeding status of the individuals are inferred with a low accuracy. The estimations of population outcrossing rates and inbreeding depression were based on the frequencies of the inbreeding classes and their average seed production, respectively. The low accuracy on inbreeding status in $\mathrm{g} 4$ is expected to inflate the confidence intervals associated with these estimations.

The population outcrossing rate between g2 and g3 $\left(t_{2}\right)$ was estimated using two different data sets. Using g3 molecular data, $t_{2}$ was 0.66 (95\% confidence interval: 0.6-0.74), whereas it reached 0.95 (0.73-0.99) using g4 molecular data. The population outcrossing rate between $\mathrm{g} 3$ and $\mathrm{g} 4\left(t_{3}\right)$ was $0.58(0.49-0.65)$.

The hermaphrodite outcrossing rates were approximated to $t_{h 2}=0.58$ and $t_{h 3}=0.42$. For the computation of $t_{h 2}$, we used the value of $t_{2}$ estimated from the g3 molecular data (see Discussion).

\section{Impact of inbreeding depression on seed production}

In g3, inbreeding significantly reduced the total seed weight and the total seed number $\left(P<10^{-5}\right)$ but did not affect seed size $(P=0.37)$. In g4, inbreeding had a significant effect on all seed production variables $\left(P<10^{-5}\right.$ for seed weight and total seed number, $P=0.02$ for seed size). In $g 3$ and $g 4$, the effect of inbreeding on seed production was independent of the competition experienced by individuals $(P>0.1$ and $P>0.4$, respectively, for all seed production variables).
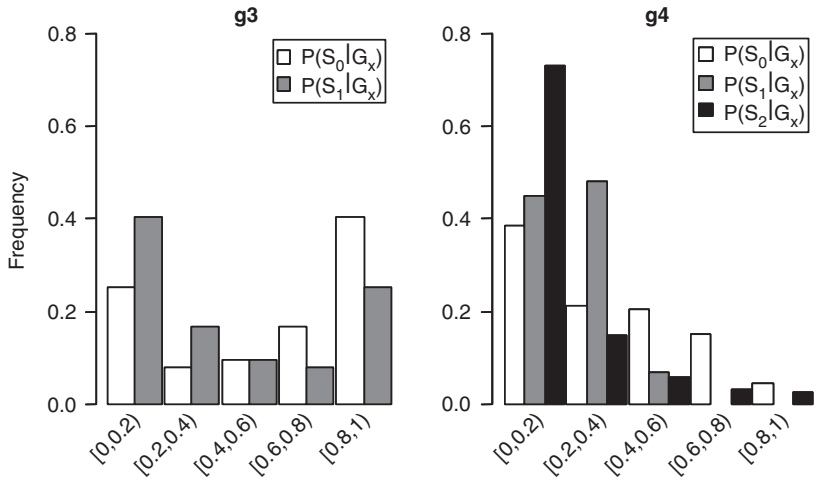

Figure 2 Distributions of the probabilities $P\left(S_{n} \mid G_{x}\right)$ in g3 and g4. $P\left(S_{n} \mid G_{x}\right)$ was computed as the probability that an individual with genotype $G_{x}$ belongs to the inbreeding class $S_{n}$. g3 and g4 contained two $\left(S_{0}\right.$ and $\left.S_{1}\right)$ and three $\left(S_{0}, S_{1}\right.$ and $S_{2}$ ) classes, respectively. Consequently, g3 individuals were characterized by the probabilities: $P\left(S_{0} \mid G_{x}\right)$ and $P\left(S_{1} \mid G_{x}\right)$, and g4 individuals by $P\left(S_{0} \mid G_{x}\right), P\left(S_{1} \mid G_{x}\right)$ and $P\left(S_{2} \mid G_{x}\right)$.

Mean values of $W_{\text {out }(g)}$ always exceeded $W_{\text {self }(g)}$ and the estimated value of inbreeding depression $\delta_{g}$ was thus always positive (Table 5). The significance tests of inbreeding depressions $\delta_{3}$ and $\delta_{4}$ were in agreement with the results of the regression model reported just above: confidence intervals did not include zero for seed weight and total seed number in $\mathrm{g} 3$ and for all seed production variables in g4. The highest value of inbreeding depression was observed for total seed number $(>21 \%)$.

The fitness reduction due to one generation of selfing did not vary between generations. In the resampled data sets consisting only of individuals belonging to inbreeding class $S_{0}$ or $S_{1}$, the proportion of data sets with a significant interaction between inbreeding class and generation was always $<5 \%$ ( $5 \%$ for seed weight, $0.1 \%$ for seed size and $4.6 \%$ for total seed number).

\section{Evolution of the frequency of females}

The frequency of females was $19 \%, 27 \%$ and $18 \%$ in g2, g3 and g4, respectively (Table 3 ). Overall, it did not vary across generations: the frequencies observed in g2 and g4 were not significantly different $\left(\chi^{2}=0.057, P=0.81\right)$.

We incorporated the parameters estimated above into the theoretical model developed by Charlesworth and Charlesworth (1978): a female advantage $(k)$ of $5 \%$, a hermaphrodite outcrossing rate $\left(t_{h}\right)$ of $50 \%$ and an inbreeding depression $(\delta)$ of $15 \%$. For these values $1+k$ did not exceed $2\left(1-\left(1-t_{\mathrm{h}}\right) \delta\right)$, which predicts that male sterility should disappear from the population. Simulations incorporating the effective population size estimate even showed that it should occur 
Table 5 Mean seed production of individuals originating from selfing and outcrossing without competition; estimated values of inbreeding depression ( $\delta$ ) and confidence intervals (Cls) in $\mathrm{g} 3$ and $\mathrm{g} 4$

\begin{tabular}{|c|c|c|c|c|c|c|}
\hline & \multicolumn{2}{|c|}{ Total seed weight (g) } & \multicolumn{2}{|c|}{ Seed size (g) } & \multicolumn{2}{|c|}{ Total seed number } \\
\hline & g3 & g4 & g3 & g4 & g3 & g4 \\
\hline$W_{\text {self }}$ & 92.86 & 114.14 & $7.88 \times 10^{-2}$ & $7.91 \times 10^{-2}$ & 1326.8 & 1769.1 \\
\hline$W_{\text {out }}$ & 114.7 & 133.12 & $7.61 \times 10^{-2}$ & $8.42 \times 10^{-2}$ & 1831.4 & 2256.6 \\
\hline$\delta$ & 0.189 & 0.142 & 0.034 & 0.06 & 0.274 & 0.215 \\
\hline $\mathrm{Cl}$ & $(0.096-0.274)$ & $(0.075-0.204)$ & $(-0.043$ to 0.106$)$ & $(0.008$ to 0.111$)$ & (0.157 to 0.373$)$ & (0.117 to 0.302$)$ \\
\hline
\end{tabular}

Table 6 Observed and simulated females frequencies in $\mathrm{g} 2, \mathrm{~g} 3$ and $\mathrm{g} 4$

\begin{tabular}{lcccc}
\hline \multirow{3}{*}{$\begin{array}{c}\text { Observed } \\
\text { values }\end{array}$} & \multicolumn{3}{c}{ Mean and confidence interval obtained by simulation } \\
\cline { 3 - 5 } & & $\begin{array}{c}t_{2}=0.66 ; t_{3}=0.58 ; \\
k=0.05 ; \delta=0.15\end{array}$ & $\begin{array}{c}t_{2}=0.66 ; t_{3}=0.58 ; \\
k=0 ; \delta=0\end{array}$ & $\begin{array}{c}t_{2}=0 ; t_{3}=0 ; \\
k=0 ; \delta=0\end{array}$ \\
\hline g2 (\%) & 19.3 & $24.8(11.6-38)$ & $25.4(12.4-38.8)$ & $25.1(13.2-38.8)$ \\
g3 (\%) & 26.9 & $18.8(6.8-31.2)$ & $18.6(6.4-33.4)$ & $16.6(5.2-31.6)$ \\
g4 (\%) & 18 & $14.9(4.4-28.8)$ & $14.5(2.8-28)$ & $12.6(2-26)$ \\
\hline
\end{tabular}

relatively quickly: out of 1000 simulated populations, 50\% did not contain females after 30 generations, and $98 \%$ after 100 generations (results not shown).

Simulations also showed that the predicted female frequencies after three generations under the alternative scenarios were almost completely overlapping (Table 6). Moreover, observed and simulated values were never significantly different.

Additional exploratory simulations performed using a larger effective population size $\left(N_{\mathrm{e}}=300\right)$, sample size $(N=1000)$ and number of generations $(g=15)$ also led to largely overlapping predictions (results not shown).

\section{DISCUSSION}

Using methods based on a joint analysis of phenotypic and multilocus genotypic data, we estimated mating system parameters in an evolving population of cultivated sunflower characterized with a nuclear determination of gynodioecy. We detected no significant resource reallocation in male-sterile individuals relative to hermaphrodites. We found that hermaphrodites were partially self-fertilizing and that inbreeding depression on seed production was between 15 and $27 \%$. Given these parameter values, theoretical models and simulations predicted that male sterility should disappear in the future generations. Below we discuss how our results contribute to document the breeding system in sunflower and how our experimental approach and study system might be of interest for studies of plant mating systems, especially the evolution of gynodioecy.

\section{Female advantage}

Taking into account numerous factors such as generation, competition and plant location, we found only a nonsignificant, slight tendency ( $\sim 5 \%)$ of females to produce more seeds than hermaphrodites. This result is not uncommon in gynodioecous species: although a female advantage was detected in most species studied, there was no or a very low impact of sexual morph on seed production in Beta vulgaris (Chenopodiaceae), Daphne laureaola (Thymelaeaceae) and Chinochloa bromoides (Poaceae; see Shykoff et al., 2003 and Dufay and Billard, 2011 for reviews). Higher levels of female advantage have been found elsewhere (Shykoff et al., 2003) and can reach values up to $100 \%$ in the few species where gynodioecy has a nuclear determinism (for instance, Fragaria virginiana, Ashman, 1999).

In gynodioecous populations, the maintenance of gender polymorphism is promoted by two main mechanisms: selection against inbreeding and resource reallocation from female compensation (a phenomenon called gender specialization in Meagher, 2007). After evaluating the relative importance of these two mechanisms in 21 gynodioecous species, Dufay and Billard (2011) concluded that for a majority of species, the avoidance of inbreeding depression could not entirely explain the female advantage. This suggests an important role for resource reallocation. In natural populations, hermaphrodites and females have coexisted for many generations, allowing the evolution of such gender specialization (Meagher, 2007). In sunflower, however, CMS and restorer genes have been introduced and artificially maintained by breeders, without any opportunity for such an adaptation to evolve.

The lack of significant sex effect on seed production does not necessarily mean an absence of resource reallocation. Indeed, females can differ from hermaphrodites in several ways that influence their fitness (Shykoff et al., 2003). Some of these traits such as seed germination ability, seedling survival and adult survival were not measured. Moreover, several studies suggest that the female advantage is environment dependent (see, for example, Asikainen and Mutikainen, 2003). For instance, if pollen limitation affected the fertilization of ovules in females (which are obligate outcrossers), females could have a lower seed production than hermaphrodites despite producing more ovules. Estimating the female advantage under more controlled environmental conditions could allow a proper investigation of the mechanisms of resource allocation to the male and female functions and to estimate the true cost of the male function. For crop species such as sunflower, this can be addressed using existing inbred lines differing only by their genotype at the restoration locus (Delph et al., 2007).

In the present study, the environmental conditions under which the seeds of the three generations were produced and the phenotypic data were collected were quite uncontrolled: density and pollination conditions varied both within and between years and there were significant spatial effects in our experimental design that affected seed production (Table 4). Thus, the absence of a significant female advantage in our experimentation indicates that it had a low impact in the real conditions where the population evolved.

\section{Outcrossing rates}

We used a method relying on multilocus genotypes to estimate outcrossing rates. Discordant estimates were obtained for the outcrossing 
rate between generation g2 and g3 using the molecular data sets from $\mathrm{g} 3$ and $\mathrm{g} 4\left(t_{2}=0.66\right.$ and 0.95 , respectively). The method of Enjalbert and David (2000) assumes no selection. However, as inbreeding depression affected the seed production of individuals from class $S_{1}$ in generation g3, the frequency of $S_{2}$ individuals in $\mathrm{g} 4$ should be impacted by selection, which is expected to bias $t_{2}$ estimates based on g4 molecular data upwards. If we exclude this last value, our estimates are concordant between generations and similar to the ones obtained in a parallel and smaller data set of the same population but using a method relying on the $F_{\mathrm{IS}}$ (Ostrowski et al., 2010). Our procedure thus seems reliable and promising. It avoids the production and analysis of progeny arrays, especially in a lowly polymorphic population such as ours, where we expect little difference in allelic composition between offspring originating from outcrossing and selfing. It is not robust to null alleles, but does not require inbreeding equilibrium. Our estimations of outcrossing rate have large confidence intervals because of the low accuracy of the breeding status inferred for each individual (Figure 2). Using a larger number of markers should improve the accuracy of this inference.

Our population estimates yielded hermaphrodite outcrossing rates of 0.58 between g2 and g3 and 0.42 between g3 and g4. These intermediate values are characteristic of animal-pollinated species (Vogler and Kalisz, 2001) and confirmed that hermaphrodite-cultivated sunflower is partially self-fertilizing in natural (uncontrolled) conditions. The disruption of the self-incompatibility system in modern varieties allowed selfing but was not sufficient for selfing to occur spontaneously. For instance, flower adaptations may be required for better self-pollen deposition, and selective pressures such as inbreeding depression may favor outcrossing over selfing (Willi and Maattanen, 2010). To our knowledge, it is the first time that the outcrossing rate effectively realized in the field was estimated for cultivated sunflower.

The selfing rate could vary as a function of the density of pollinators (Degrandi-Hoffman and Chambers, 2006) or of pollen availability, influenced for instance by sex ratio (Miyake and Olson, 2009), and could evolve in response to these factors (see, for example, Willi and Maattanen, 2010). Investigating the phenotypic plasticity of the selfing rate and the factors affecting its variability and evolution would be interesting in sunflower and more generally to better understand mating systems evolution. It is especially meaningful in the context of global pollinator declines (Eckert et al., 2010) and in the search for a lower dependency on pollinators for seed production in sunflower (Degrandi-Hoffman and Chambers, 2006).

\section{Inbreeding depression}

Inbreeding significantly affected seed production variables in all generations. Inbreeding and consequently inbreeding depression should increase as the population approaches inbreeding equilibrium. To get a rough estimate of the inbreeding depression expected at equilibrium, we (1) computed the mean fitness of individuals belonging to the inbreeding class $S_{n}$, assuming that fitness decreased linearly with increasing inbreeding coefficient and (2) used an iterative process to predict the equilibrium frequencies of the inbreeding classes with a population outcrossing rate of 0.5 . Equilibrium inbreeding depression was approximated to $19 \%$ for seed weight. This value is very close to the inbreeding depression observed in g3 and g4 (18.9\% and $14.2 \%$, respectively) but is somewhat lower than values typically observed in outcrossing species ( $\sim 28 \%$; Husband and Schemske, 1996). Interestingly, our estimate is lower than the value of $50 \%$ needed to allow the maintenance of outcrossing in the population (Lande and Schemske, 1985). We must remind that we ignored inbreeding depression at the juvenile stage as well as on pollen production. Moreover, our population derives from a F1-hybrid variety that results from the cross of two inbred lines: the inbreeding load of such plant material has already been purged by breeders, decreasing the expected inbreeding depression. No data are yet available to compare our estimates with the inbreeding depression existing in old landraces and in wild populations of the species.

Inbreeding effect could decrease across generations, thanks to the purge of the genetic load (Husband and Schemske, 1996), but no decrease was detected in our study. The selfing history of the parental inbred lines (see above), the short time scale of the experiment, the small effective population size and the moderate levels of self-fertilization (Glemin, 2003) could all contribute to impede the detection of any purge of the genetic load.

In the literature, the impact of competition on inbreeding depression is not clearcut. Several studies have reported that inbreeding depression increased under stronger competition (Dudash, 1990), whereas others, such as ours, found no evidence of this phenomena (for example, Hebe subalpina; Delph and Lloyd, 1996). As proposed by Norman et al. (1995), this could be explained if competition impedes growth to such a degree that differences between inbred and outcrossed progeny remain hidden.

\section{Predicted evolution of the population}

Taking into account both selective and stochastic processes, we showed that the persistence of male sterility is unlikely after 100 generations (probability of observing females $<5 \%$ ). The disappearance of females, despite the importance of stochastic processes $\left(N_{\mathrm{e}}<40\right)$, is not surprising. Indeed, with hermaphrodites outcrossing at a rate of 0.5 , females are expected to be maintained in the population only if $(k+\delta) \geqslant 1$, and our estimates give $(k+\delta)=0.2$. As discussed above, these parameters were estimated based on phenotypic data collected at the adult stage only. However, the discrepancy between the estimated values and the required condition makes relatively improbable that additional data at the juvenile stage could reverse our expectations for the persistence of male sterility.

We analyzed three generations of the experimental population: in such a short time scale, and with such low population size, it is again not surprising that the frequency of females did not vary significantly and that different evolutionary scenarios led to largely overlapping predictions.

\section{Potentialities of our system for the study of mating system evolution}

Simple models with estimable parameters are useful for the study of mating system evolution. Our experimental design seems promising, even if not powerful enough to validate theoretical expectations:

- It conforms to simple models of gynodioecy evolution, and allows the estimation of all parameters affecting its evolution. In addition to what is mentioned above (that is, incorporation of the juvenile stage and phenotypic plasticity assessment), our understanding of the determinism of restoration remains incomplete, especially of the level of dominance of the nuclear restorer allele. Visual observations suggest that in some individuals the restoration of the male function is only partial, as observed before, for instance, in B. vulgaris (Dufay et al., 2008).

- It can be associated with methods of inference relying on genotyping and phenotyping a sample of the generation, without the need of producing particular progenies (such as selfed vs outcrossed progenies, or progeny arrays for outcrossing rate estimation). 
Parameters can be estimated in the conditions where the generations have been produced.

- It can benefit from molecular tools available or under development for this crop species, such as the mapping of the restoration locus and numerous molecular markers (Yue et al., 2010).

With an appropriate design (that is, replicates and well-chosen environmental conditions; measurements ranging over the whole life cycle), short-scale experiments can tackle long-standing questions, such as the impact of population and environmental context on parameter values. If conducted for further generations, a manipulation of the selective pressures will be needed to observe a significant evolution (see, for example, David and Pham, 1993). Finally, natural populations of weedy sunflowers, fixed for the male-sterile cytoplasm and submitted to recurrent gene flow from cultivated fields (bringing recurrently Rf and rf alleles, Muller et al., 2011), could constitute an interesting case study in relation with models including gene flow in the evolution of gynodioecy (Dufay and Pannell, 2010).

\section{DATA ARCHIVING}

Data have been deposited at Dryad: doi:10.5061/dryad.6t779.

\section{CONFLICT OF INTEREST}

The authors declare no conflict of interest.

\section{ACKNOWLEDGEMENTS}

We thank Alain Gil and Véronique Viader for technical assistance and Joëlle Ronfort, Laurène Gay and two anonymous referees for critical review of the manuscript. We also thank the INRA Experimental Unit of Epoisses (UE115) and Xavier Reboud for the production of the first two generations of the population.

Ashman TL (1999). Determinants of sex allocation in a gynodioecious wild strawberry: implications for the evolution of dioecy and sexual dimorphism. J Evol Biol 12: 648-661.

Asikainen E, Mutikainen P (2003). Female frequency and relative fitness of females and hermaphrodites in gynodioecious Geranium sylvaticum (Geraniaceae). Am J Bot 90: 226-234.

Belkhir K, Borsa P, Chikhi L, Raufaste N, Bonhomme F (2001). GENETIX 4.02, logiciel sous Windows ${ }^{\mathrm{TM}}$ pour la génétique des populations. Laboratoire Génome, Populations, Interactions, CNRS UMR 5000, Université de Montpellier II: Montpellier, France.

Charlesworth B, Charlesworth D (1978). Model for evolution of dioecy and gynodioecy. Am Nat 112: 975-997.

David JL, Pham JL (1993). Rapid changes in pollen production in experimental outcrossing populations of wheat. J Evol Biol 6: 659-676.

Degrandi-Hoffman G, Chambers M (2006). Effects of honey bee (Hymenoptera: Apidae) foraging on seed set in self-fertile sunflowers (Helianthus annuus L.). Env Ent 35: 1103-1108.

Delph LF, Lloyd DG (1996). Inbreeding depression in the gynodioecious shrub Hebe subalpina (Scrophulariaceae). New Zeal J Bot 34: 241-247.

Delph LF, Touzet P, Bailey MF (2007). Merging theory and mechanism in studies of gynodioecy. Trends Ecol Evol 22: 17-24.

Dudash MR (1990). Relative fitness of selfed and outcrossed progeny in a self-compatible, protandrous species, Sabatia angularis L. (Gentianaceae): a comparison in three environments. Evolution 44: 1129-1139.

Dufay M, Billard E (2011). How much better are females? The occurrence of female advantage, its proximal causes and its variation within and among gynodioecious species. Ann Bot (e-pub ahead of print 31 March 2011; doi:10.1093/aob/mcr062).

Dufay M, Pannell JR (2010). The effect of pollen versus seed flow on the maintenance of nuclear-cytoplasmic gynodioecy. Evolution 64: 772-784.

Dufay M, Vaudey V, De Cauwer I, Touzet P, Cuguen J, Arnaud J-F (2008). Variation in pollen production and pollen viability in natural populations of gynodioecious Beta vulgaris ssp. maritima: evidence for a cost of restoration of male function? J Evol Biol 21: $202-212$.
Eckert CG, Kalisz S, Geber MA, Sargent R, Elle E, Cheptou PO et al. (2010). Plant mating systems in a changing world. Trends Ecol Evol 25: 35-43.

Ellstrand NC, Heredia SM, Leak-Garcia JA, Heraty JM, Burger JC, Yao L et al. (2010). Crops gone wild: evolution of weeds and invasives from domesticated ancestors. Evol App/ 3: 494-504.

Enjalbert J, David J (2000). Inferring recent outcrossing rates using multilocus individual heterozygosity: application to evolving wheat populations. Genetics 156: 1973-1982.

Frankham R (1995). Effective population size/adult population size ratios in wildlife: a review. Genet Res 66: 95-107.

Gandhi SD, Heesacker AF, Freeman CA, Argyris J, Bradford K, Knapp SJ (2005). The selfincompatibility locus (S) and quantitative trait loci for self-pollination and seed dormancy in sunflower. Theor Appl Genet 111: 619-629.

Glemin S (2003). How are deleterious mutations purged? Drift versus non random mating. Evolution 57: 2678-2687.

Glémin S, Bataillon T (2009). A comparative view of the evolution of grasses under domestication. New Phytol 183: 273-290.

Goldringer I, Bataillon T (2004). On the distribution of temporal variation in allele frequency: consequences for the estimation of effective population size and the detection of loci undergoing selection. Genetics 168: 563-568.

Goldringer I, Prouin C, Rousset M, Galic N, Bonnin I (2006). Rapid differentiation of experimental populations of wheat for heading time in response to local climatic conditions. Ann Bot 98: 805-817.

Gross BL, Olsen KM (2010). Genetic perspectives on crop domestication. Trends Plant Sci 15: 529-537.

Hancock JF (2005). Contributions of domesticated plants studies to our understanding of plant evolution. Ann Bot 96: 953-963.

Husband BC, Schemske DW (1996). Evolution of the magnitude and timing of inbreeding depression in plants. Evolution 50: 54-70.

Lande R, Schemske DW (1985). The evolution of self-fertilization and inbreeding depression in plants. I. Genetic models. Evolution 39: 24-40.

Meagher TR (2007). Linking the evolution of gender variation to floral development. Ann Bot 100: 165-176.

Miyake K, Olson MS (2009). Experimental evidence for frequency dependent selffertilization in the gynodioecious plant, Silene Vulgaris. Evolution 63: 1644-1652.

Muller M-H, Latreille M, Tollon C (2011). The origin and evolution of a recent agricultural weed: population genetic diversity of weedy populations of sunflower (Helianthus annuus L.) in Spain and France. Evol App/ 4: 499-514.

Nei M (1987). Molecular Evolutionary Genetics. Columbia University Press: New York.

Nielsen R, Mattila DK, Clapham PJ, Palsboll PJ (2001). Statistical approaches to paternity analysis in natural populations and applications to the North Atlantic humpback whale. Genetics 157: 1673-1682.

Norman JK, Sakai AK, Weller SG, Dawson TE (1995). Inbreeding depression in morphological and physiological traits of Schiedea lydgatei (Caryophyllaceae) in two environments. Evolution 49: 297-306.

Ostrowski MF, Rousselle Y, Tsitrone A, Santoni S, David J, Reboud X et al. (2010). Using linked markers to estimate the genetic age of a volunteer population: a theoretical and empirical approach. Heredity 105: 358-369.

Putt ED (1997). Early history of sunflower. In: Schneiter AA (ed). Sunflower Technology and Production. ASA, CSSA, SSSA: Madison, WI, pp 1-19.

R Development Core Team (2008). R: A Language and Environment for Statistical Computing. R Foundation for Statistical Computing: Vienna, Austria, ISBN 3900051-07-0.http://www.R-project.org.

Rieseberg LH, Van Fossen C, Arias DM, Carter RL (1994). Cytoplasmic male sterility in sunflower: origin, inheritance, and frequency in natural populations. $J$ Hered 85: 233-238.

Ross-Ibarra J, Morrell PL, Gaut BS (2007). Plant domestication: a unique opportunity to identify the genetic basis of adaptation. Proc Natl Acad Sci USA 104(Suppl 1): 8641-8648.

Shykoff JA, Kolokotronis SO, Collin CL, Lopez-Villavicencio M (2003). Effects of male sterility on reproductive traits in gynodioecious plants: a meta-analysis. Oecologia 135: $1-9$.

Siol M, Bonnin I, Olivieri I, Prosperi JM, Ronfort J (2007). Effective population size associated with self-fertilization: lessons from temporal changes in allele frequencies in the selfing annual Medicago truncatula. J Evol Biol 20: 2349-2360.

Tang S, Yu JK, Slabaugh MB, Shintani DK, Knapp SJ (2002). Simple sequence repeat map of the sunflower genome. Theor App/ Genet 105: 1124-1136.

Vogler DW, Kalisz S (2001). Sex among the flowers: the distribution of plant mating systems. Evolution 55: 202-204.

Waples RS (1989). A generalized approach for estimating effective population size from temporal changes in allele frequency. Genetics 121: 379-391.

Willi Y, Maattanen K (2010). Evolutionary dynamics of mating system shifts in Arabidopsis lyrata. J Evol Biol 23: 2123-2131.

Wolfram S (1996). The Mathematica Book, 3rd edn. Wolfram Media/Cambridge University Press: Cambridge, UK

Yue B, Vick BA, Cai X, Hu J (2010). Genetic mapping for the Rf1 (fertility restoration) gene in sunflower (Helianthus annuus L.) by SSR and TRAP markers. Plant Breed 129: 24-28. 


\section{APPENDIX}

At each generation of selfing, the heterozygosity resulting from the initial outcrossing event is halved. Thus, if an individual $x$ has been genotyped at $L$ unlinked polymorphic loci, the probability of its multilocus heterozygosity pattern $G_{x}$ given its inbreeding class is (Enjalbert and David, 2000):

$$
P\left(G_{x} \mid x \in S_{n}\right)=\prod_{l=1}^{L}\left(\frac{H_{e_{l}}}{2^{n}}\right)^{a_{l}}\left(1-\frac{H_{e_{l}}}{2^{n}}\right)^{1-a_{l}},
$$

where $H_{e l}$ is Nei's gene diversity at locus $l$ (Nei, 1987) and provides the probability of being heterozygous after an outcrossing event and $a_{l}$ is a binary variable: $a_{l}=1$ if $x$ is heterozygous at locus $l$ and $a_{l}=0$ otherwise.

According to Bayes theorem, at a given generation, the probability that an individual with genotype $G_{x}$ belongs to class $S_{n}$ can be written as:

$$
P\left(x \in S_{n} \mid G_{x}\right)=\frac{P\left(G_{x} \mid x \in S_{n}\right) \times P\left(x \in S_{n}\right)}{P\left(G_{x}\right)},
$$

where $P\left(x \in S_{n}\right)$ corresponds to the frequency of individuals belonging to class $S_{n}$, and $P\left(G_{x}\right)$ to the probability of $G_{x}$, the genotype of individual $x$ :

$$
P\left(G_{x}\right)=\sum_{n=0}^{2} P\left(G_{x} \mid x \in S_{n}\right) P\left(x \in S_{n}\right) .
$$

$P\left(x \in S_{n}\right)$ is a function of the population outcrossing rates of the past generations. Namely, $P\left(x \in S_{1}\right)$ is $\left(1-t_{2}\right)$ in g3 and $\left(1-t_{3}\right)$ in g4. $P\left(x \in S_{2}\right)$ is $\left(1-t_{2}\right)\left(1-t_{3}\right)$ in g4. Thus, $P\left(x \in S_{n} \mid G_{x}\right)$ can be obtained by integrating equation (A2) on the distribution of $\left(t_{2}, t_{3}\right)$.
For individuals in g3:

$$
P\left(x \in S_{n} \mid G_{x}\right)=\int_{0}^{1} P\left(x \in S_{n} \mid G_{x}, t_{2}\right) f\left(t_{2}\right) \mathrm{d} t_{2},
$$

where the density $f\left(t_{2}\right)$ approximates the true discrete distribution of $t_{2}$.

In the absence of any information on $f\left(t_{g}\right)$, we assumed uniform distributions between 0 and 1 . Then, we updated these distributions using the information on population outcrossing rate available in the genetic data from all the $Y$ individuals of generation $g$, following Nielsen et al. (2001). For individuals in g3, the probability $P\left(x \in S_{n} \mid G_{x}\right)$ becomes:

$$
\begin{aligned}
P(x \in & \left.S_{n} \mid G_{x}\right) \\
& =\frac{\int_{0}^{1} P\left(x \in S_{n} \mid G_{x}, t_{2}\right) \prod_{y, y \neq x} P\left(G_{y} \mid t_{2}\right) f\left(t_{2}\right) \mathrm{d} t_{2}}{\int_{0}^{1} \prod_{y} P\left(G_{y} \mid t_{2}\right) f\left(t_{2}\right) \mathrm{d} t_{2}} .
\end{aligned}
$$

As a practical approach, we used the approximation proposed by Nielsen et al. (2001):

$$
P\left(x \in S_{n} \mid G_{x}\right)=\int_{0}^{1} P\left(x \in S_{n} \mid G_{x}, t_{2}\right) \omega \mathrm{d} t_{2},
$$

where

$$
\omega=\frac{\prod_{y} P\left(G_{y} \mid t_{2}\right) f\left(t_{2}\right)}{\int_{0}^{1} \prod_{y} P\left(G_{y} \mid t_{2}\right) f\left(t_{2}\right) \mathrm{d} t_{2}} .
$$

We computed these probabilities by numerically integrating equation (A6) on a grid containing 500 points. As a simplification, these individual probabilities $P\left(x \in S_{n} \mid G_{x}\right)$ are denoted $P\left(S_{n} \mid G_{x}\right)$ in the following. 